\title{
A GENERALIZATION OF MALLIAVIN'S UNIQUENESS THEOREM ${ }^{* \dagger \ddagger}$
}

\author{
GuANTIE DENG
}

\begin{abstract}
Usıng Malliavin's uniqueness theorem about Watson's Problem, we obtain a generalization of Malliavın's uniqueness results and a discrete version of a PhragménLindelöf theorem.
\end{abstract}

\section{Introduction}

Recently, B. Korenblum and the others give some results about a generalization of Carleman's uniqueness theorem and a discrete Phragmén-Lindelöf theorem. In this paper, we will give a further generalization about these results by using a generalization of Malliavin's uniqueness theorem.

Let $v(x)$ be a function defined on $[0,+\infty)$ and let $H(v)$ be the set of such functions $f(z)$ which are holomorphe in the half-plane $C_{+}=\{z=x+i y: x>0\}$ continuous in the closed half-plane $\operatorname{cl}\left(C_{+}\right)=\{z=x+i y: x \geq 0\}$ such that the following condition

$$
|f(z)| \leq A \exp \{A x+x v(x)\}
$$

hold for $z=x+i y \in C_{+}, r=|z|$. (The symbol $A$ is used for the large enough, positive constant, not necessarily the same at each occurrence.) Let $\Lambda=\left\{\lambda_{n}\right\}$ be an increasing sequence of positive real numbers such that the following separation condition

$$
8 \delta=\inf \left\{\lambda_{n+1}-\lambda_{n}: n=1,2, \ldots\right\}>0
$$

and the following Malliavin's uniqueness condition ([11]) for $H(v)$

$$
\int_{1}^{\infty} S(\Lambda(r)-a) r^{-2} d r=+\infty
$$

holds for any real number $a$, where

\footnotetext{
† 1991 Mathematics Subject Classification: 30E05, 26E10.

$\ddagger$ Key words: Phragmén-Lindelöf Theorem, Malliavın’s Unıqueness theorem, Watson’s Problem

* The project supported by the National Natural Science Foundation of China

Received June 14, 1999; revised January 19, 2000.
} 


$$
\begin{gathered}
\Lambda(r)=2 \sum_{\lambda \leq r, \lambda \in \Lambda} \frac{1}{\lambda} \quad \text { if } r \geq \lambda_{1}, \quad \text { and } \Lambda(r)=0, \quad \text { if } r<\lambda_{1} ; \\
S(t)=\sup \{x t-x v(x): x \geq 0\} .
\end{gathered}
$$

Malliavin's uniqueness theorem ([8] and [11]) says that, if $f \in H(v)$ and $f(\lambda)=0$ for $\lambda \in \Lambda,(2)$ and (3) hold, then $f \equiv 0$ on $C_{+}$. Therefore we shall call the set $\Lambda$, which satisfies (2) and (3), Malliavin's uniqueness set for $H(v)$. A well-known Phragmén-Lindelöf Theorem says that if $f \in H(1)$ is bounded in the positive real axis, then $f$ is bounded in $\operatorname{cl}\left(\boldsymbol{C}_{+}\right)$. In this paper, we prove that if the separation condition (2) and Malliavin's uniqueness condition (3) hold, $f \in H(v)$ and

$$
\rho=\varlimsup_{n \rightarrow+\infty} \lambda_{n}^{-1} \log \left|f\left(\lambda_{n}\right)\right|<\infty
$$

then $f$ is of exponential type $\rho$. So we write our theorem as follows:

THEOREM 1. Suppose that the set $\Lambda=\left\{\lambda_{n}\right\}$ is Malliavin's uniqueness set for $H(v)$. If $f \in H(v)$ and (5) holds, then $f$ is of exponential type $\rho$ and

$$
|f(z)| \leq A \exp (\rho x)
$$

holds for $z=x+i y \in \boldsymbol{C}_{+}$.

Remark 1. If $\rho=-\infty$ then $f \equiv 0$, so our theorem is a generalization of Malliavin's uniqueness theorem.

As a corollary of Theorem 1, we have the following theorem about a signed Borel measure.

THEOREM 2. Suppose that the set $\Lambda=\left\{\lambda_{n}\right\}$ is Malliavin's uniqueness set for $H(v)$. If $\mu$ is a signed Borel measure on $(-\infty,+\infty)$ and

$$
\begin{gathered}
\int_{-\infty}^{+\infty} e^{t x}|d \mu(t)| \leq A \exp (x v(x)+x A) \quad \text { for } x>0, \\
\rho=\varlimsup_{n \rightarrow+\infty} \lambda_{n}^{-1} \log \left(\int_{-\infty}^{+\infty} e^{\lambda_{n} t}|d \mu(t)|\right)<\infty
\end{gathered}
$$

then $d \mu$ is a measure supported on $(-\infty, \rho]$.

\section{Proof of Theorems}

In order to prove Theorem 1, we need a generalization of Malliavin's uniqueness theorem about Watson's problem ([4], [5], [6] and [11]).

LEMMA 1. Let $\Lambda=\left\{\lambda_{n}\right\}$ be a sequence of positive numbers such that (2) holds, let $v(x)$ be a continuous, increasing function on $[0,+\infty)$ and let $\Lambda(r)$ be defined by (4). Suppose that the function $g(z)$ is analytic in $\boldsymbol{C}_{+}$, continuous in 
$\operatorname{cl}\left(\boldsymbol{C}_{+}\right)$such that

$$
|g(z)| \leq 1+\exp \{x v(x)-x \Lambda(r)+A x\} .
$$

If (3) holds, then $g(z)$ is bounded in $C_{+}$and the upper boundedness is not greater than 2.

The proof of Lemma 1 is similar to that given in [5], [6], [8], [11] and is here omitted.

Proof of Theorem 1. W. H. J. Fuchs ([8]) has proved that the function $v$ in (1) can be replaced by a continuous, increasing function and the uniqueness condition (3) also satisfy. So we suppose that such conditions also hold, we suppose also that $\lambda_{1} \geq 8 \delta$ hold. First the function

$$
f_{1}(z)=\frac{f(z)}{G(z)(1+z)^{2}}
$$

is analytic in $C_{+}-\Lambda$, where $G(z)$ is Fuch's function ([7] and [12]) defined by

$$
G(z)=\prod_{n=1}^{\infty}\left(\frac{z-\lambda_{n}}{z+\lambda_{n}}\right) \exp \left(\frac{2 z}{\lambda_{n}}\right) .
$$

W. H. J. Fuchs ([7] and [12]) has proved that the function $G(z)$ is analytic in the half plane $\left\{z=x+i y: x>-\lambda_{1}\right\}$, and that

$$
\begin{gathered}
|G(z)| \leq \exp \{x \Lambda(r)+A x\}, \quad z \in C_{+}, \quad r=|z| \\
|G(z)| \geq \exp \{x \Lambda(r)-A x\}, \quad z \in C(\Lambda, \delta) \\
\left|G^{\prime}\left(\lambda_{n}\right)\right| \geq \exp \left\{\lambda_{n} \Lambda\left(\lambda_{n}\right)-A \lambda_{n}\right\}, \quad n=1,2, \ldots,
\end{gathered}
$$

where $C(\Lambda, \delta)=C_{+}-\bigcup_{n=1}^{+\infty} D\left(\lambda_{n}, \delta\right), D\left(\lambda_{n}, \delta\right)=\left\{z:\left|z-\lambda_{n}\right| \leq \delta\right\}$. Therefore we obtain that

$$
\left|f_{1}(z)\right| \leq \frac{A}{1+|z|^{2}} \exp \{x v(x)-x \Lambda(r)+A x\}
$$

holds for $z \in C(\Lambda, \delta), r=|z|$. The function $h_{2}(t)$ defined by

$$
h_{2}(t)=-\frac{1}{2 \pi i} \int_{-l \infty}^{+l \infty} f_{1}(\zeta) e^{-\zeta t} d \zeta
$$

is continuous on $(-\infty,+\infty)$ and that

$$
h_{2}(t)=\sum_{\lambda \in \Lambda, \lambda<\xi} a(\lambda) e^{-\lambda t}-\frac{1}{2 \pi i} \int_{\xi-l \infty}^{\xi+l \infty} f_{1}(\zeta) e^{-\zeta t} d \zeta
$$

holds for $\xi>0, \xi \notin \Lambda$, where the coefficients $a(\lambda)$ are the residues of $f_{1}(z)$ at the points $\lambda \in \Lambda$. Since 


$$
a(\lambda)=\frac{f(\lambda)}{G^{\prime}(\lambda)(1+\lambda)^{2}} ; \quad \lambda \in \Lambda, \quad \lim _{n \rightarrow+\infty} \frac{\log \left|a\left(\lambda_{n}\right)\right|}{\lambda_{n}}=-\infty,
$$

(Malliavin's Uniqueness condition (3) implies that $\Lambda(r)$ is unbounded in $[0, \infty)$.) the function

$$
h_{3}(t)=\sum_{\lambda \in \Lambda} a(\lambda) e^{-\lambda t}
$$

is an entire function of $t=\sigma+i \tau$. The function $g_{\sigma}(z)$ defined by

$$
g_{\sigma}(z)=\int_{\sigma}^{+\infty}\left(h_{3}(t)-h_{2}(t)\right) \exp \{z(t-\sigma)\} d t
$$

is an entire function of $z=x+i y$, and for $z \notin \Lambda, x<\xi, \xi \notin \Lambda$

$$
g_{\sigma}(z)=-\frac{1}{2 \pi i} \int_{\xi-l \infty}^{\xi+l \infty} \frac{f_{1}(\zeta)}{\zeta-z} e^{-\zeta \sigma} d \zeta-\sum_{\lambda<\xi, \lambda \in \Lambda} \frac{a(\lambda)}{\lambda-z} e^{-\lambda \sigma}
$$

and for $x>0$,

$$
g_{\sigma}(z)=-\frac{1}{2 \pi i} \int_{-l \infty}^{+l \infty} \frac{f_{1}(\zeta)}{\zeta-z} e^{-\zeta \sigma} d \zeta-\sum_{\lambda \in \Lambda} \frac{a(\lambda)}{\lambda-z} e^{-\lambda \sigma}-f_{1}(z) e^{-z \sigma}
$$

since, for any $\xi>0, \xi \notin \Lambda$ and any $\sigma$, there exists a constant $A(\xi, \sigma), \lambda_{\xi}=$ $\inf \{\lambda: \lambda \in \Lambda, \lambda>\xi\}$ such that

$$
\left|h_{3}(t)-h_{2}(t)\right| \leq A(\xi, \sigma)\left[\exp (-\xi t)+\exp \left\{-\lambda_{\xi}(t-\sigma)\right\}\right] .
$$

So there exists a constant $B(\sigma)$ depending only on $\sigma$, and $\delta$ such that, for $x \leq 4 \delta$, $z=x+i y$, we have

$$
\left|g_{\sigma}(z)\right| \leq \frac{B(\sigma)}{6 \delta-x}
$$

and for $x \geq 0$, we have

$$
\left|g_{\sigma}(z)\right| \leq A(\sigma)+\exp \{A+A \sigma-x \sigma+x v(x)-x \Lambda(|z|)\}
$$

where $A(\sigma)$ is a constant depending only on $\sigma$. Lemma implies that the function $g_{\sigma}(z)$ is bounded in the entire complex plane, so it follows from the Liouville theorem that the entire function $g_{\sigma}(z)$ is identically equal to a constant, thus the entire function $g_{\sigma}(z)$ is identically equal to zero. Therefore the following equality

$$
f_{1}(z) \exp (-z \sigma)=-\frac{1}{2 \pi l} \int_{-l \infty}^{+\infty \infty} \frac{f_{1}(\zeta)}{\zeta-z} \exp (-\zeta \sigma) d \zeta-\sum_{\lambda \in \Lambda} \frac{a(\lambda)}{\lambda-z} \exp (-\lambda \sigma)
$$

holds for $z=x+i y, x>0$ and for any $\sigma$. By taking $\sigma=-\Lambda(r)+A$ in (9), we obtain, from (5) and (2), that

$$
|f(z)| \leq A+A \sum_{\lambda_{n} \leq r} \exp \left(\lambda_{n}\left[\Lambda(r)-\Lambda\left(\lambda_{n}\right)\right]\right)+A \sum_{\lambda_{n} \geq r} \exp \left(-\lambda_{n}\right) \leq A \exp (A r)
$$


(Using $|\Lambda(x)-\Lambda(y)| \leq A|\log x-\log y|, \quad$ and $\sup \left\{t(\log r-\log t: t>0\}=r e^{-1}\right.$ ) where $r=|z|$. Therefore the function $f(z)$ is of exponential type in the half plane, bounded on the imaginary axis by (1), so a well-known discrete PhraménLindelöf theorem ([1] p. 200 and [2]) implies that (6) holds. by

Proof of Theorem 2. The condition (7) implies that the function $f(z)$ defined

$$
f(z)=\int_{-\infty}^{+\infty} e^{t z} d \mu(t)
$$

is analytic in $\boldsymbol{C}_{+}=\{z=x+i y: x>0\}$ continuous in the closed half-plane $\left.\operatorname{cl}\left(\boldsymbol{C}_{+}\right)\right)$ $=\{z=x+i y: x \geq 0\}$ and the conditions (1) and (5) are satisfied. Theorem 1 implies that (6) holds.

Define the function $F(z)$ by setting

$$
F(z)=\frac{f(z)}{1+z} e^{-\rho z}
$$

Clearly, $F$ is analytic on $C_{+}=\{z=x+i y: x>0\}$ continuous in the closed halfplane $\left.\operatorname{cl}\left(C_{+}\right)\right)=\{z=x+i y: x \geq 0\}$ and is square summable on the imaginary axis. Thus we can apply the Paley-Wiener theorem ([13], p. 8, Theorem V) to concludde that

$$
F(z)=\int_{-\infty}^{0} \psi(t) e^{t z} d t
$$

for some $\psi \in L^{2}((-\infty, 0))$. We shall assume that $\psi$ is defined for all real numbers (by setting $\psi(t)=0$ for all $t>0$ ).

On the imaginary axis, we have two representation for $F$

$$
\int_{-\infty}^{+\infty} \psi(t) e^{i y t} d t=F(i y)=\frac{e^{-\rho l y}}{1+i y} \int_{-\infty}^{+\infty} e^{i t y} d \mu(t)
$$

Using notation $\tilde{f}$ for the Fourier transform of $f$ (i.e., $\tilde{f}(x)=\int_{-\infty}^{+\infty} f(y) e^{l y x} d y$ ), and letting, $\psi_{\rho}(t)=\psi(t-\rho)$, we arrive at

where $\gamma$ is the function

$$
\widetilde{\psi_{\rho}}(y)=\tilde{\gamma}(y) \widetilde{d \mu}(y)
$$

$$
\gamma(t)=e^{t} \quad \text { if } t \leq 0 ; \quad \text { and } \quad \gamma(t)=0 \quad \text { if } t>0
$$

Hence

$$
\psi_{\rho}(y)=\psi(y-\rho)=(\gamma * d \mu)(y)=\int_{-\infty}^{+\infty} \gamma(y-x) d \mu(x)=\int_{y}^{+\infty} e^{y-x} d \mu(x)
$$

Thus, for all $t>\rho$, we have $\int_{t}^{+\infty} e^{-x} d \mu(x)=0$, which implies that the total variation of $d \mu$ on $(\rho,+\infty)$ is 0 ; i.e., the measure $d \mu(t)$ is supported in $(-\infty, \rho]$. This complete the proof of Theorem 2. 
Acknowledgments. The author would like to express his gratitude to Professor Paul Malliavin for his hospitality and his invitation to visit Université Pierre et Marie Curie.

\section{REFERENCES}

[1] R. P Boas, JR., Entire Functions, Academic Press, New York, 1954.

[2] J. B. Conway, Functins of One Complex Variable, Springer-Verlag, 1973.

[3] G. T. Deng, Uniqueness of some holomorphic functions, Chiness Ann. Math. Ser. B, 7 (1986), 330-338.

[ 4 ] G. T. DENG, On Watson's problem and its applicatıons, Bull. Sc1. Math. 2., 109 (1985), 4-15.

[ 5 ] G. T. DeNG, une condition nécessaire et suffisante pour la quasi-analyticité de Mandelbrojt sur une demi-droite, C. R. Acad. Scı, Parıs I Math., 306 (1988), 769-772.

[6] G. T. Deng, Théorème d'existence et d'unicité pour les fonctions méromorphes dans un dem1plan, Bull. Sc1. Math. 2., 113 (1989), 443-462.

[ 7] W H. J. Fuchs, On the closure of $\left\{e^{-t} t^{a_{i}}\right\}$, Proc. Cambridge Philos. Soc. 42 (1946), 91-105.

[8] W H. J. Fuchs, An application of the Ahlfors distortion theorem, J. Analyse Math., 18 (1976), 61-79.

[ 9 ] B. Korenblum, A. Mascuilli, and J. Panariello, A generalization of Carleman's uniqueness theorem and a discrete Phragmén-Lindelöf Theorem, Proc. Amer. Math. Soc., 126 (1998), 2025-2032.

[10] P Malliavin, Sur la croissance radiale d'une fonction Méromorphe, Illinois J. Math., 1 (1957), 179-255.

[11] P Malliavin, Sur quelques procédés d'extrapolation, Acta Math., 83 (1955), 179-255.

[12] S. Mandelbrojt, Séries adhérentes, Régularısatıon des suites, Applicatıons, Gauthır-Villars, Paris, 1952.

[13] R. Paley and N. Wiener, Fourier Transforms in the Complex Damain, Colloquium Publications, 19, American Mathematical Society, Providence, 1934.

DePartment OF Mathematics

BEIJING NORMAL UNIVERSITY

100875 BeIJING

P.R. ChINA 